Adapting the Body Appreciation Scale-2 for Children: A Psychometric Analysis of the BAS-2C

\author{
Emma Halliwell, Hannah Jarman, \\ Centre for Appearance Research, University of the West of England, UK; \\ Tracy Tylka, \\ Ohio State University, USA \\ Amy Slater, \\ Centre for Appearance Research, University of the West of England, UK
}

Author Note

Emma Halliwell, Centre for Appearance Research, University of the West of England, UK;

Hannah Jarman, Centre for Appearance Research, University of the West of England, UK; Tracy Tylka, Ohio State University, USA;

Amy Slater, Centre for Appearance Research, University of the West of England, UK

Address correspondence: Emma Halliwell, Centre for Appearance Research, Department of Psychology, University of the West of England, Frenchay, Coldharbour Lane, Bristol, BS16 9QY. E-mail: emma.halliwell@ uwe.ac.uk. Phone: +44 (0)117 3282154 


\begin{abstract}
Positive body image among adults is related to numerous indicators of well-being. However, no research has explored body appreciation among children. To facilitate our understanding of children's positive body image, the current study adapts and validates the Body Appreciation Scale-2 (BAS-2; Tylka \& Wood-Barcalow, 2015a) for use with children. Three hundred and forty-four children (54.4\% girls) aged 9-11 completed the adapted Body Appreciation Scale-2 for Children (BAS-2C) alongside measures of body esteem, media influence, body surveillance, mood, and dieting. A sub-sample of 154 participants (62.3\% girls) completed the questionnaire 6-weeks later to examine stability (test-retest) reliability. The BAS-2C displayed a unidimensional factor structure and evidence of internal consistency, test-retest reliability, and construct, criterion-related, and incremental validity. Additionally, the results suggest adaptive properties of body appreciation for body-related and emotional well-being among children. The BAS-2C could serve as an essential component within research to understand and estimate children's positive body image.
\end{abstract}

Keywords: children, Body Appreciation Scale-2, positive body image, assessment, psychometrics, well-being, preadolescents 


\section{Adapting the Body Appreciation Scale-2 for Children: A Psychometric Analysis of the}

\section{BAS-2C}

Over the last decade, the emergence of positive body image research represents an important shift from a primary focus on disturbances to a broader exploration of the body image concept (Smolak \& Cash, 2011; Tylka, 2011; Tylka \& Wood-Barcalow, 2015b). Research demonstrates that, among adults, positive body image is a more comprehensive construct than the absence of negative body image (Tylka \& Wood-Barcalow, 2015b) and is associated with numerous and diverse indicators of well-being (Avalos, Tylka, \& WoodBarcalow, 2005; Tylka \& Kroon Van Diest, 2013; Tylka \& Wood-Barcalow, 2015a; Wasylkiw, MacKinnon, \& MacLellan, 2012). Moreover, initial levels of positive body image predicted future levels of adaptive (i.e., intuitive) eating among 12- to 16-year-old girls (Andrew, Tiggemann, \& Clark, 2016).

While first generation measures of positive body image, such as the Body Esteem Scale (Mendelson, Mendelson, \& White, 2001), assess a global positive evaluation of the body, second generation measures of positive body image tap into a more complex and nuanced understanding of this construct (Webb, Butler-Ajibade, \& Robinson, 2014). The most widely used second generation measure of positive body image is the Body Appreciation Scale (BAS; Avalos et al., 2005), which is validated for use with adult women and men (Avalos et al., 2005; Tiggemann, 2015; Tylka \& Wood-Barcalow, 2015a). To date no research has examined positive body image among children using a second generation measure, given that such a measure has not yet been validated for use with children. Recently, scholars have highlighted the development of such a measure as a priority (Halliwell, 2015; Webb, Wood-Barcalow, \& Tylka, 2015), as an age-appropriate measure must first be developed to be able to begin research that would facilitate our understanding of positive body image among children, including its development, maintenance, and fluctuation across time. 
Adapting an existing second generational measure of positive body image, such as the Body Appreciation Scale (Avalos et al., 2005; Tylka \& Wood-Barcalow, 2015a), for use with children would be a useful step towards this goal. The original BAS is a unidimensional 13item scale concentrated around four key highly interrelated concepts of positive body image: favourable opinions of one's body, body acceptance, respect of one's body, and rejection of narrow appearance ideals promoted by the media (Avalos et al., 2005). Participants rate their level of agreement on a 5-point Likert-type scale ranging from Never (1) to Always (5). All items are positively worded, and one item varies depending on the gender of the participant. Whereas the BAS demonstrated excellent psychometric support among college women (Avalos et al, 2005) and men (Tylka, 2013), it is limited because the gender-specific item necessitates separate forms for women and men, it contains body dissatisfaction-based language (e.g., using terms such as "flaws"), and its unidimensional factor structure does not replicate in certain cultures. To address these limitations, Tylka and Wood-Barcalow (2015a) revised the gender-specific item to be gender-neutral, removed any assumption that individuals view their bodies as imperfect, and deleted items that consistently exhibited item-factor loadings $<.50$ both in Western and non-Western cultures. Finally, they added five items to the remaining five original items to capture advances in knowledge in the positive body image literature garnered since the publication of the original BAS. The resulting slightly modified scale, the BAS-2, assesses a single factor incorporating body acceptance, appreciating the functionality of the body, caring for the body, and the influence of inner positivity on outer demeanor. Tylka and Wood-Barcalow (2015a) found support for the psychometric properties of the BAS-2 among college and community samples of US men and women, including high internal consistency $(\alpha$ s $=.93-.96)$ and stability over a 3-week period $(r=.90)$.

Since the development of the BAS, positive body image research has advanced and diversified. The BAS-2 has now been validated across several cultures and adult populations, including Dutch (Alleva, Martijn, Veldhuis, \& Tylka, 2016), Iranian (Atari, 2016), Hong Kong 
(Swami \& Ng, 2015), and Chinese (Swami, Ng, \& Barron, 2016) samples. The body appreciation latent construct has been found to be equivalent across U.S. men and women (Tylka, 2013; Tylka \& Wood-Barcalow, 2015a), allowing for gender comparisons in BAS-2 scores. Moreover, the BAS-2 has shown good internal reliability among 14 and 15-year-old girls (Halliwell et al., 2015). As such, it would be useful to determine whether the BAS-2 can also be used to conceptualize and assess children's positive body image.

Theoretically, we would expect children to experience high levels of positive body image if they receive messages of body acceptance from others, with the acknowledgement that negative environmental factors such as appearance-related pressures (e.g., pressures to be thin and/or muscular) have the potential to disrupt this positive experience (Halliwell, 2015; Piran \& Teall, 2013). A validated measure of positive body image for preadolescents is essential to investigate the developmental trajectory of body image among children, which may in turn help inform, facilitate, and develop appropriate body image interventions (Halliwell, 2015; Webb et al., 2015). Therefore, the aim of this study is to adapt and validate the BAS-2 for use among preadolescent children. First, we conducted a pilot study to assess the face validity, comprehensibility, and acceptability of the measure items for children. Second, we explored the factor structure and garnered reliability estimates for the Body Appreciation Scale-2 for Children (BAS-2C). In addition, construct (i.e., convergent), criterion-related, and incremental validity of the BAS-2C was assessed through its associations with other measures of body image, mood, and dieting. We hypothesized that the BAS-2C would reveal a unidimensional factor structure and its scores would be internally consistent and stable over time. In addition, we predicted that the BAS-2C would demonstrate construct (convergent) validity via its relations with body-related variables (body esteem, media influence, body surveillance) and mood, show evidence of criterion-related (concurrent) validity via its associations with dieting, and reveal incremental validity via its unique contributions to mood after accounting for its shared variance in body esteem. 


\section{Method}

\section{Participants}

The total sample consisted of 344 children (54.4\% girls) aged between 9 and 11 years $\left(M_{\mathrm{age}}=9.34, S D_{\mathrm{age}}=0.69\right)$ who were recruited from four primary schools in the South-West of England. The majority of schools were larger than the average U.K. primary school in size, had a below average proportion of students eligible for free school meals, and had an above average proportion of students with a disability or special educational needs. Participant ethnicity was not queried; however, all schools contained a majority of White British students.

Two schools were randomly selected and approached to complete the BAS-2C on a second occasion, six weeks after the first administration, which provided a large enough sample to gauge test-retest reliability ( $n=154,62.3 \%$ girls) without collecting unnecessary data from young participants. Participants who provided data on both occasions were aged between 9 and 11 years $\left(M_{\mathrm{age}}=9.98, S D_{\mathrm{age}}=0.69\right)$. There were no significant differences between the participants that were and were not included in the retest administration on the two variables assessed across time: age, $t(309)=-1.09, p=.276, d=-0.12$, and body appreciation, $t(308)=-1.64, p=.105, d=-0.17$.

\section{Measures}

Demographics. Participants reported their age, gender, school, and school year.

Body appreciation. We adapted the 10-item Body Appreciation Scale-2 (BAS-2; Tylka \& Wood-Barcalow, 2015a) for children. To do this, three boys and three girls (all White, 9-11 years old) were recruited into a pilot study through the authors' personal contacts. The aim of this pilot was to identify any issues related to understanding or appropriateness of the BAS-2 items for this younger cohort. Participants were interviewed individually and were encouraged to talk openly. Each participant was provided with the 10 BAS-2 items which they were instructed not to complete. The authors facilitated the interviews by reading each item aloud to the participant. For each item participants were asked "Does this statement make 
sense to you?", "Do you think this statement would make sense to other children your age", "Could you respond to this statement on the Never, Seldom, Sometimes, Often, Always scale?", and "Do you think we need to make any changes to this statement?" Researchers noted participants' responses to these open-ended questions.

Items and the response scale were revised according to this feedback; in total, four items were revised for clarity. The original BAS-2 Item 4, "I am attentive to my body's needs" was modified to "I pay attention to what my body needs." The original BAS-2 Item 7, "I appreciate the different and unique characteristics of my body" was slightly revised to, "I appreciate the different and unique things about my body." The original BAS-2 Item 8, "My behavior reveals my positive attitude toward my body; for example, I hold my head high and smile," was modified to, "You can tell I feel good about my body by the way I behave." The original BAS-2 Item 10, "I feel like I am beautiful even if I am different from media images of attractive people (e.g., models, actresses/actors)" was altered to, "I feel like I am beautiful even if I am different from pictures and videos of attractive people (e.g., models/actresses/actors)." Additionally, the presentation order of the first and second BAS-2 items was reversed (i.e., the original BAS-2 Item 1 is Item 2 in the BAS-2C, and the original BAS-2 Item 2 is Item 1 in the BAS-2C), as this arrangement better set the tone of the scale for children. In terms of item response options, the 5-point scale was retained; however, the response option Seldom in the BAS-2 was replaced with Rarely in the BAS-2C, as children thought that Rarely was easier to understand. Thus, the response scale in the BAS-2C was: Never (scored as 1), Rarely (2), Sometimes (3), Often (4), and Always (5).

Body esteem. The Appearance subscale of the Body Esteem Scale for Children (BESC; Mendelson \& White, 1993) was used to measure appearance-related body esteem. This subscale consists of 13 items (e.g., "I like what I look like in pictures") which are rated on a 5point Likert scale from No-disagree a lot (scored as 1) to Yes - agree a lot (5). Originally, the BES-C utilised a yes or no response scale; however, it was adapted to a 5-point scale to 
improve sensitivity. Items are averaged, with higher scores indicating greater body esteem. Scores on this subscale have yielded internal consistency and moderate 2-week test-retest reliability with children as young as eight (Vander Wal \& Thelen, 2000). In the present study, Cronbach's alphas for appearance-related body esteem were .89 for boys and .94 for girls. These coefficients are comparable to alpha coefficients reported in other research with children, e.g., $\alpha=.92$ (Vander Wal \& Thelen, 2000).

Media influence. The Multidimensional Media Influence Scale (MMIS; Cusumano \& Thompson, 2001) contains three subscales: Awareness (three items, e.g., "Clothes look better on people who are thin"), Internalization (six items, e.g., 'I try to look like the models in magazines"), and Pressure of media influences (two items, e.g., "Watching TV or reading magazines makes me want to diet or lose weight"). Items are rated on a 5-point Likert scale from $\mathrm{No}$-disagree a lot (scored as 1) to Yes - agree a lot (5). Items were averaged within their respective subscales, with higher subscale scores representing greater media influence. Scores on the MMIS have demonstrated evidence of internal consistency among children aged 8-11 years (Cusumano \& Thompson, 2001). In the present study, Cronbach's alphas for Awareness, Internalization, and Pressure, respectively, were $.76, .87$, and .76 for boys and $.79, .89$, and .78 for girls. These coefficients are comparable to those reported by Cusumano and Thompson (2001) for Awareness (.73), Internalization (.91), and Pressure (.71).

Body surveillance. The Body Surveillance subscale of the Objectified Body Consciousness Scale-Youth (OBCS-Y; Lindberg, Hyde, \& McKinley, 2006) was used to measure the extent to which children monitor their physical appearance. The scale consists of four items (e.g., "During the day, I think about how I look many times") which are rated on a 5-point Likert scale from No-disagree a lot (scored as 1) to Yes - agree a lot (5). All items are averaged, with higher scores indicating greater body surveillance. This subscale has been found to yield internally consistent and stable scores over a 2-week period among girls aged 912 years (Lindberg et al., 2006). In the present study, Cronbach's alphas for Body Surveillance 
were .80 for boys and .86 for girls. These coefficients are comparable to the alpha of .88 reported by Lindberg et al. (2006) for Body Surveillance.

Mood. The Positive and Negative Affect Schedule for Children (PANAS-C; Ebesutani et al., 2012) was used to measure positive and negative affect. Participants were asked to rate how much they have felt five positive emotions (e.g., "Joyful") and five negative emotions (e.g., "Scared") in the past few weeks on a 5-point Likert-type scale ranging from Not at all (scored as 1) to Extremely (5). Positive and negative emotion items were averaged separately to provide subscale scores, with higher scores indicating a greater tendency to experience either positive or negative mood. Scores on these subscales have demonstrated evidence of internal consistency among children aged 6-18 years (Ebesutani et al., 2012). In the present study, Cronbach's alphas for Positive Affect and Negative Affect, respectively, were .74 and .71 for boys and .80 and .84 for girls. These are comparable to the Cronbach alpha coefficients reported by Ebesutani et al. (2012) for Positive Affect (.86) and Negative Affect (.85).

Dieting behaviours. Two items gauged participants' current and previous dieting behaviors: "Are you currently dieting to lose weight?" and "Have you dieted to lose weight in the last year?" Participants could choose No (0) or Yes (scored as 1).

\section{Procedure}

The study was approved by the university ethics committee at XXX (blinded for review). Four local primary schools were recruited. Passive parental consent (in which parents could opt-out their child from the research) and active participant assent were obtained. Neither schools nor participants received incentives to participate. Participants completed the questionnaires in their classrooms. One researcher read each questionnaire item aloud (participants then responded to each question anonymously on their questionnaire). A second researcher was available to respond to questions and issues. This method of reading questions aloud is commonly used and recommended in research with children to accommodate a range of reading and comprehension ability (e.g., McCabe, Connaughton, Tatangelo, Mellor, \& 
Busija, 2017). However, reading questions aloud meant that counterbalancing was not possible within classes and all participants completed the measures in the same order: body esteem, media influence, surveillance, body appreciation, mood, dieting, and age. A practice item 'I like swimming" was presented at the beginning of the questionnaire, and this item was discussed to make sure that all children understood the response options. The questionnaires took approximately 20 minutes to complete. This same procedure was followed 6 weeks later to provide test-retest data from a sub-sample of participants; the BAS-2C was the only scale administered at Time 2. Previous research has indicated that 6 weeks is appropriate to assess stability in body image measures (Wertheim, Paxton, \& Tilgner, 2004).

\section{Results}

\section{Preliminary Analyses}

Across all samples, the count for individual missing data points (i.e., all scale items across all participants) was very low, with $1.14 \%$ missing over all outcome data. According to Little's MCAR analyses, these data were missing completely at random, $\chi^{2}(1674)=1713.96, p$ $=.244$. Bennet (2001) indicates analyses are prone to bias if more than $10 \%$ of the data are missing. Therefore, we decided not to impute data and the analyses are reported using pairwise deletion. Data screening did not show any unduly influential observations for any variables; thus, no univariate or multivariate outliers were deleted. Means and standard deviations for all study variables are reported in Table 2 . There were gender differences in study variables for body appreciation, $t(309)=1.97, p=.050$, body esteem, $t(309)=4.22, p<.001$, body surveillance, $t(309)=-4.13, p<.001$, and negative affect, $t(309)=-2.67, p=.004$, with boys scoring higher than girls on body appreciation and body esteem and lower than girls on body surveillance and negative affect.

\section{Factor Structure}

Exploratory factor analysis was conducted separately by gender, consistent with the validation of the BAS-2 (Tylka \& Wood-Barcalow, 2015a). Principal axis factor analysis with 
quartimax rotation, the orthogonal rotation procedure of choice when a general factor is expected (Pedhazur \& Schmelkin, 1991), was conducted to allow for the possibility of more than one factor. Data were appropriate for factor analys is, as the Bartlett's test of sphericity was significant for both girls' data, $\chi^{2}(45)=761.41, p<.001$, and boys' data, $\chi^{2}(45)=546.98$, $p<.001$, indicating that the variables are interrelated and factorable, and the Kasier-MeyerOlkin $(\mathrm{KMO})$ test indicated that the sample was compact for both girls' data, $\mathrm{KMO}=.90$, and boys' data, $\mathrm{KMO}=.91$. Only one eigenvalue greater than 1.0 emerged for both samples; this single factor explained $53.78 \%$ of the item variance in girls' data and $51.26 \%$ of the variance in boys' data. All factor loadings are reported in Table 1 and exceeded the minimum level required for retention of .32, as recommended by Tabachnick and Fidell (2007).

\section{Internal Consistency Reliability}

Cronbach's coefficient alphas for the BAS-2C were .89 in the full sample $(n=124)$ and .88 in the retest sample of boys $(n=36)$, and .90 in the full sample $(n=147)$ and .89 in the retest sample of girls $(n=71)$. For boys and girls, respectively, item-total correlations ranged between .48-.73 and .39-.76. These values support the internal consistency of the BAS-2C.

\section{Construct Validity}

It was hypothesized that the BAS-2C would be related to established body image measures. The BAS-2C was strongly positively correlated with body esteem, $r=.76, p<.001$, (95\% CI .71, .80) and moderately-to-strongly negatively correlated with body surveillance, $r=$ $-.61, p<.001$ (95\% CI -.66, -.54), awareness of media influence, $r=-.30, p<.001,(95 \% \mathrm{CI}-$ $.40,-.20)$, internalization of media influence, $r=-.45, p<.001$ (95\% CI -.54, -.36), and media influence pressures, $r=-.49, p<.001(95 \% \mathrm{CI}-.57,-.40)$. Table 2 presents these relationships separated by gender. These results support the convergent validity of the BAS-2C.

\section{Criterion-related Validity}

As hypothesized, the BAS-2C was positively related to positive affect, $r=.57, p<.001$ $(95 \%$ CI $.49, .64)$ and inversely related to negative affect, $r=-.35, p<.001$ (95\% CI -.44, - 
.25). Body appreciation was significantly lower among girls who were currently dieting $(M=$ 3.54, $S D=0.88)$ compared to girls who were not currently dieting $(M=3.91, S D=0.76)$, $t(162)=-2.57, p=.010, d=0.45$. Similarly, body appreciation was significantly lower among girls who had previously dieted $(M=3.54, S D=0.85)$ than girls who had not previously dieted $(M=3.95, S D=0.76), t(163)=-3.05, p=.003, d=0.50$. For boys, however, there was no significant difference on body appreciation between current dieters $(M=4.02, S D=0.97)$ and non-dieters $(M=4.00, S D=0.72), t(136)=0.13, p=.890, d=0.02$. However, boys who had previously dieted reported lower body appreciation $(M=3.72, S D=1.00)$ than boys who had not previously dieted $(M=4.12, S D=0.66), t(135)=-2.65, p=.009, d=-0.47$. These findings add support for the BAS-2C's criterion-related validity.

\section{Incremental Validity}

To examine whether the BAS-2C explained additional variance in mood over and above body esteem, a series of regressions were conducted. Body esteem was entered at Step 1, and body appreciation was entered at Step 2 .

Positive affect. For girls, Step 1 was significant, $F(1,167)=81.51, p<.001$, and the addition of body appreciation at Step 2 was also significant, $\beta=.53, p<.001, \Delta F(1,166)=$ $27.48, p<.001$, contributing $10 \%$ of unique variance in positive affect $\left(\Delta R^{2}=.10\right)$ above its shared contribution with body esteem. For boys, Step 1 was significant, $F(1,139)=69.78, p<$ .001 . However, the addition of body appreciation at Step 2 was not significant, $\beta=.13, p=$ $.179, \Delta F(1,138)=1.82, p=.179$.

Negative affect. For girls, Step 1 was significant, suggesting that body esteem accounted for a significant proportion of the variance in negative affect, $F(1,167)=77.41, p<$ .001 , yet the addition of body appreciation did not significantly increase the amount of explained variance, $\beta=-.06, p=.59, \Delta F(1,166)=.00, p=.589$. For boys, Step 1 was significant, $F(1,139)=17.56, p<.001$, and the addition of body appreciation at Step 2 was 
marginally significant, $\beta=.21, p=.056, \Delta F(1,138)=3.72, p=.056$, accounting for $2 \%$ of unique variance in negative affect $\left(\Delta R^{2}=.02\right)$ above its shared contribution with body esteem. Test-retest Reliability

Intraclass correlation coefficients (ICCs) and paired samples $t$-tests were used to assess test-retest reliability of the BAS-2C among the sub sample of 154 participants who completed the measure twice, approximately 6 weeks apart. The ICC for boys' data was $.81, p<.001$ ( $95 \% \mathrm{CI} .65, .89)$, and the ICC for girls' data was also .81, $p<.001$ (95\% CI .71, .88).

Furthermore, BAS-2C scores did not change significantly over time for boys (Time $1 M=$ 4.24, $S D=0.63$; Time $2 M=4.30, S D=0.67), t(44)=-0.83, p=.414, d=-0.09$, or for girls (Time $1 M=3.91, S D=0.76$; Time $2 M=4.01, S D=0.84$ ), $t(80)=-1.38, p=.170, d=-0.12$. Therefore, BAS-2C scores were stable over time for girls and boys.

\section{Discussion}

Given the importance of studying positive body image development among children, we converted a psychometrically sound measure of positive body image in adults, the BAS-2 (Tylka \& Wood-Barcalow, 2015a), to be appropriate for children as young as age 9 to complete. To achieve this goal, a pilot sample of children (three boys and three girls aged 911) evaluated the BAS-2 items and response scale for clarity, appropriateness, and understanding. This evaluation resulted in retaining the original 10 BAS-2 items, with slight modifications to four items, revising one of the points along the original 5-point response scale (i.e., Rarely replaced Seldom), and switching the order of the first two items to better set the tone of the scale for a younger sample. The resultant scale, the BAS-2C, was found to have a unidimensional factor structure in a large sample of 9- to 11-year-old children. Moreover, scores on the BAS-2C were internally consistent and stable, as well as demonstrated construct and criterion-related validity, in this young sample. The BAS2-C is included in the Appendix. Based on the current findings, we recommend that this version of the BAS-2 items and response options are used with children. In addition, to facilitate comprehension of the scale 
and to be consistent with the administration of other validated measures among children, we recommend that items are read aloud and that children are given the opportunity to ask questions about anything they do not understand.

Importantly, these findings illustrated the adaptive properties of body appreciation for the body-related and emotional well-being of children. While the adaptive properties of body appreciation have been noted for adolescents, college students, and adults (Andrew et al., 2016; Tylka \& Wood-Barcalow, 2015a), it was not clear how early body appreciation is linked to well-being in children prior to the findings of the present study. Our findings suggest that, for both girls and boys, higher body appreciation is associated with higher appearance-related body esteem and positive affect, and is inversely related to body surveillance, internalization of media influence, and previous and current dieting behaviours. In addition, high body appreciation among girls is also inversely related to awareness of media influe nce, pressure to look like media appearance ideals, and negative affect.

Our findings further attest to the distinction between body appreciation, a second generation measure of positive body image, and body esteem, a first generation measure. Whereas the Body Esteem Scale assesses generalised positive feelings toward the body, the BAS-2 taps into body acceptance, caring for the body and appreciation of body function. Indeed, the BAS-2C was superior to body esteem in explaining variance in positive affect among girls and, more marginally, negative affect among boys.

\section{Limitations and Future Research}

It is important to interpret the present study's findings in light of its limitations. First, we did not collect data on ethnicity, although we do know the majority of students in the schools sampled were White. Thus, our findings cannot generalize to ethnically diverse children. Furthermore, we did not collect data on families' socioeconomic status, and our age range (9-11 years) was relatively narrow and older than the typical age at which internalization of media appearance ideals begin (i.e., as young as 3 years old; Harriger, Calogero, 
Witherington, \& Smith, 2010). Researchers need to explore whether the BAS-2C evidences a unidimensional factor structure and reliable and valid scores among children of various age, ethnic, and socioeconomic groups.

Second, the BAS-2C is limited in that it is self-report, relying on children accurately portraying their attitudes toward their body. We did not include an estimate of their socially desirable responding; such an estimate could help determine if they are consciously or unconsciously trying to project a favourable yet inaccurate evaluation of their bodies. Further research is necessary to determine whether the BAS-2C is related to impression management, as well as other indices used to estimate discriminant validity.

Third, this study was correlational in design and therefore no inferences can be made about the directionality between body appreciation, media influence, body surveillance, and dieting behaviours. It is imperative to conduct longitudinal research on body appreciation among children to determine its ability to predict future levels of adaptive and maladaptive attitudes and behaviors. Researchers also need to investigate how body appreciation transitions over time during different developmental stages, while also considering how social identities (e.g., race, gender, sexual orientation, socioeconomic status, and physical abilities) shape experiences of bodies during these stages. Another related area of research is exploring how body appreciation may be protective by offsetting body image threats (e.g., appearance-related pressures, puberty) among children throughout development.

Fourth, we assessed body appreciation's unique associations with only one well-being index (i.e., mood) after accounting for its shared variance in appearance-related body esteem. Further research is needed to explore the relative role of body appreciation in explaining incremental variance in other aspects of children's physical and psychological well-being, such as self-esteem and intuitive eating.

\section{Conclusion}


The present study illustrated that the BAS-2 can be adapted to a younger sample of children with relatively few modifications, while maintaining the psychometric integrity of its scores. Its brevity, and the ease of completing and scoring, will make the BAS-2C an attractive option (a) to include in correlational, prospective, and experimental research on children's body appreciation as well as (b) to integrate when investigating the efficacy and effectiveness of prevention programs. Thus, the BAS-2C could serve as an essential component within research to understand and estimate children's positive body image, which is a fertile and important area greatly in need of attention (Smolak \& Cash, 2011). 


\section{References}

Alleva, J. M., Martijn, C., Veldhuis, J., \& Tylka, T. L. (2016). A Dutch translation and validation of the Body Appreciation Scale-2: An investigation with female university students in the Netherlands. Body Image, 19, 44-48. doi: 10.1016/j.bodyim.2016.08.008

Andrew, R., Tiggemann, M., \& Clark, L. (2016). Predictors and health-related outcomes of positive body image in adolescent girls: A prospective study. Developmental Psychology, 52, 463-474. doi: 10.1037/dev0000095

Atari, M. (2016). Factor structure and psychometric properties of the Body Appreciation Scale2 in Iran. Body Image, 18, 1-4. doi:10.1016/j.bodyim.2016.04.006

Avalos, L. C., Tylka, T. L., \& Wood-Barcalow, N. (2005). The Body Appreciation Scale: Development and psychometric evaluation. Body Image, 2, 285-297. doi: 10.1016/j.bodyim.2005.06.002

Bennet, D. A. (2001). How can I deal with missing data in my study? Australian and New Zealand Journal of Public Health, 25, 464-469. doi:10.111/j.1467842x.2001.tb00294.x

Cusumano, D. L., \& Thompson, J. K. (2001). Media influence and body image in 8-11-yearold boys and girls: A preliminary report on the Multidimensional Media Influence Scale. International Journal of Eating Disorders, 29, 37-44. doi: 10.1002/1098108X(200101)29:1<37::AID-EAT6>3.0.CO;2-G

Ebesutani, C., Regan, J., Smith, A., Reise, S., Higa-McMillan, C., \& Chorpita, B. F. (2012). The 10-item Positive and Negative Affect Schedule for Children, child and parent shortened versions: Application of item response theory for more efficient assessment. Journal of Psychopathology and Behavioral Assessment, 34, 191-203. doi: $10.1007 / \mathrm{s} 10862-011-9273-2$

Halliwell, E. (2015). Future directions for positive body image research [Special series]. Body Image, 14, 177-189. doi: 10.1016/j.bodyim.2015.03.003 
Halliwell, E., Jarman, H., McNamara, A., Risdon, H., \& Jankowski, G. (2015). Dissemination of evidence-based body image interventions: A pilot study into the effectiveness of using undergraduate students as interventionists in secondary schools. Body Image, 14, 1-4. doi:10.1016/jlbodyim.2015.02.002

Harriger, J. A., Calogero, R. M., Witherington, D. C., \& Smith, J. E. (2010). Body size stereotyping and internalization of the thin ideal in preschool girls. Sex Roles, 63, 609620. doi: $10.1007 / \mathrm{s} 11199-010-9868-1$

Lindberg, S. M., Hyde, J. S., \& McKinley, N. M. (2006). A measure of objectified body consciousness for preadolescent and adolescent youth. Psychology of Women Quarterly, 30, 65-76. doi: 10.1111/j.1471-6402.2006.00263.x

McCabe, M. P., Connaughton, C., Tatangelo, G., Mellor, D., \& Busija, L. (2017). Healthy me: A gender-specific program to address body image concerns and risk factors among preadolescents. Body Image, 20, 20-30. doi: 10.1016/j.bodyim.2016.10.007

Mendelson, B. K., \& White, D. R. (1993). Manual for the Body-Esteem Scale for Children. Concordia University Research Bulletin, 12, 1-10.

Pedhazur, E. J., \& Schmelkin, L. P. (1991). Measurement, design, and analysis: An integrated approach. Hillsdale, NJ: Lawrence Erlbaum.

Piran, N., \& Teall, T. (2013). The developmental theory of embodiment. In G. McVey, M. P. Levine, N. Piran, \& H. B. Ferguson (Eds.), Preventing eating-related and weightrelated disorders (pp. 169-197). Ontario, Canada: Wilfrid Laurier University Press.

Smolak, L. (2011). Body image development in childhood. In T. F. Cash \& L. Smolak, Body image: A handbook of science, practice, and prevention ( $2^{\text {nd }}$ ed., pp. 67-75). New York: Guilford.

Smolak, L., \& Cash, T. F. (2011). Future challenges for body image science, practice, and prevention. In T. F. Cash \& L. Smolak (Eds.), Body image: A handbook of science, practice, and prevention (2nd ed., pp. 471-478). New York: Guilford Press. 
Swami, V., \& Ng, S.-K. (2015). Factor structure and psychometric properties of the Body Appreciation Scale-2 in university students in Hong Kong. Body Image, 15, 68-71. doi: 10.1016/j.bodyim.2015.06.004

Swami, V., Ng, S.-K., \& Barron, D. (2016). Translation and psychometric evaluation of a Standard Chinese version of the Body Appreciation Scale-2. Body Image, 18, 23-26. doi: 10.1016/j.bodyim.2016.04.005

Tabachnick, B. G., \& Fidell, L. S. (2007). Using multivariate statistics ( $5^{\text {th }}$ ed.). Boston, MA: Allyn \& Bacon.

Tiggemann, M. (2015). Considerations of positive body image across various social identities and special populations [Special series]. Body Image, 14, 168-176. doi: 10.1016/j.bodyim.2015.03.002

Tylka, T. L. (2011). Positive psychology perspectives on body image. In T. F. Cash \& L. Smolak (Eds.), Body image: A handbook of science, practice, and prevention ( $2^{\text {nd }}$ ed., pp. 56-64). New York: Guilford Press.

Tylka, T. L. (2013). Evidence for the Body Appreciation Scale's measurement equivalence/invariance between U.S. college women and men. Body Image, 10, 415418. doi: 10.1016/j.bodyim.2013.02.006

Tylka, T. L., \& Kroon Van Diest, A. M. (2013). The Intuitive Eating Scale-2: Item refinement and psychometric evaluation with college women and men. Journal of Counseling Psychology, 60, 137-153. doi: 10.1037/a0030893

Tylka, T. L., \& Wood-Barcalow, N. L. (2015a). The Body Appreciation Scale: Item refinement and psychometric evaluation. Body Image, 12, 53-67. doi: 10.1016/j.bodyim.2014.09.006

Tylka, T. L., \& Wood-Barcalow, N. L. (2015b). What is and what is not positive body image? Conceptual foundations and construct definition [Special series]. Body Image, 14, 118129. doi: 10.1016/j.bodyim.2015.04.001 
Vander Wal, J. S., \& Thelen, M. H. (2000). Predictors of body image dissatisfaction in elementary-age school girls. Eating Behaviors, 1, 105-122. doi: 10.1016/S14710153(00)00011-8

Wasylkiw, L., MacKinnon, A. L., \& MacLellan, A. M. (2012). Exploring the link between self-compassion and body image in university women. Body Image, 9, 236-245. doi: 10.1016/j.bodyim.2012.01.007

Webb, J. B., Wood-Barcalow, N. L., \& Tylka, T. L. (2015). Assessing positive body image: Contemporary approaches and future directions [Special series]. Body Image, 14, 130145. doi: 10.1016/j.bodyim.2015.03.010 


\section{Appendix}

Body Appreciation Scale 2 - Children (BAS-2C)

This set of questions asks what you think of your body. The options are 'Never,' 'Rarely,' 'Sometimes,' Often,' and 'Always.' Please circle the word which shows how often you feel this way.

1. I feel good about my body.

2. I respect my body.

3. I feel that my body has at least some good qualities.

4. I take a positive attitude towards my body.

5. I pay attention to what my body needs.

6. I feel love for my body.

7. I appreciate the different and unique things about my body.

8. You can tell I feel good about my body by the way I behave.

9. I am comfortable in my body.

10. I feel like I am beautiful even if I am different from pictures and videos of attractive people (e.g. models/actresses/actors). 
Table 1

BAS-2C Items, Means, Standard Deviations, and Item-Factor Loadings among Girls and Boys

\begin{tabular}{|c|c|c|c|c|c|}
\hline & & $\begin{array}{c}\text { Girls } \\
M(S D)\end{array}$ & $\begin{array}{l}\text { Factor } \\
\text { loading }\end{array}$ & $\begin{array}{c}\text { Boys } \\
M(S D)\end{array}$ & $\begin{array}{l}\text { Factor } \\
\text { loading }\end{array}$ \\
\hline 1. & I feel good about my body & $3.57(1.16)$ & .79 & $3.95(1.13)$ & .78 \\
\hline 2. & I respect my body & $4.18(1.04)$ & .79 & $4.27(1.02)$ & .65 \\
\hline 3. & $\begin{array}{l}\text { I feel that my body has at least some } \\
\text { good qualities }\end{array}$ & $3.96(1.08)$ & 69 & $4.24(1.05)$ & .68 \\
\hline 4. & $\begin{array}{l}\text { I take a positive attitude towards my } \\
\text { body }\end{array}$ & $3.80(1.06)$ & .69 & $4.12(0.98)$ & .79 \\
\hline 5. & I pay attention to what my body needs & $4.30(0.87)$ & .37 & $4.35(0.85)$ & .48 \\
\hline 6. & I feel love for my body & $3.67(1.19)$ & .76 & $3.86(1.25)$ & .75 \\
\hline 7. & $\begin{array}{l}\text { I appreciate the different and unique } \\
\text { things about my body }\end{array}$ & $3.97(1.09)$ & .77 & $4.10(1.05)$ & .71 \\
\hline 8. & $\begin{array}{l}\text { You can tell I feel good about my body } \\
\text { by the way I behave }\end{array}$ & $3.21(1.26)$ & .45 & 3.49 (1.28) & .55 \\
\hline & I am comfortable in my body & $3.94(1.12)$ & .80 & $4.33(1.04)$ & .77 \\
\hline & $\begin{array}{l}\text { I feel like I am beautiful even if I am } \\
\text { different from pictures and videos of } \\
\text { attractive people (e.g., } \\
\text { models/actresses/actors) }\end{array}$ & $3.77(1.25)$ & .75 & $3.45(1.39)$ & .56 \\
\hline
\end{tabular}

Note. Girls $n=187$, boys $n=157$. 
Table 2

Variable Means, Standard Deviations, and Correlations among Girls and Boys

\begin{tabular}{|c|c|c|c|c|c|c|c|c|c|c|c|}
\hline & $\begin{array}{c}\text { Girls } \\
M(S D)\end{array}$ & $\begin{array}{c}\text { Boy } \\
M(S D)\end{array}$ & 1 & 2 & 3 & 4 & 5 & 6 & 7 & 8 & 9 \\
\hline 1 Age & $\begin{array}{l}9.96 \\
(0.71)\end{array}$ & $\begin{array}{l}9.88 \\
(0.67)\end{array}$ & & $-.17^{*}$ & $-.19^{*}$ & -.01 & -.04 & .06 & .04 & -.13 & $.20^{*}$ \\
\hline 2 Body appreciation & $\begin{array}{l}3.84^{a} \\
(0.80)\end{array}$ & $\begin{array}{l}4.01^{\mathrm{a}} \\
(0.77)\end{array}$ & -.12 & & $.67^{* * *}$ & -.14 & -.05 & $-.21 *$ & $-.20 *$ & $.46 * * *$ & -.11 \\
\hline 3 Body esteem & $\begin{array}{l}3.59^{c} \\
(0.93)\end{array}$ & $\begin{array}{l}4.00^{c} \\
(0.74)\end{array}$ & -.04 & $.81^{* * *}$ & & -.13 & -.15 & $-.34 * * *$ & $-.33 * * *$ & $.58^{* * *}$ & $-.34^{* * *}$ \\
\hline 4 Awareness & $\begin{array}{l}2.82 \\
(1.10)\end{array}$ & $\begin{array}{l}2.71 \\
(1.07)\end{array}$ & .03 & $-.38 * * *$ & $-.41^{* * *}$ & & $.45^{* * *}$ & $.24 * *$ & $.43 * * *$ & -.13 & .08 \\
\hline 5 Internalization & $\begin{array}{l}2.06 \\
(1.01)\end{array}$ & $\begin{array}{l}1.85 \\
(0.97)\end{array}$ & -.06 & $-.45^{* * *}$ & $-.59 * * *$ & $.53 * * *$ & & $.39 * * *$ & $.63 * * *$ & -.04 & $.22^{* *}$ \\
\hline 6 Pressure & $\begin{array}{l}2.22 \\
(1.19)\end{array}$ & $\begin{array}{l}2.05 \\
(1.15)\end{array}$ & -.04 & $-.49 * * *$ & $-.57^{* * *}$ & $.46 * * *$ & $.65^{* * *}$ & & $.51 * * *$ & $-.19 *$ & .04 \\
\hline 7 Body surveillance & $\begin{array}{l}2.85^{c} \\
(1.24)\end{array}$ & $\begin{array}{l}2.29^{c} \\
(1.09)\end{array}$ & .02 & $-.55^{* * *}$ & $-.70^{* * *}$ & $.37^{* * *}$ & $.67^{* * *}$ & $.48^{* * *}$ & & $-.22^{*}$ & $.27^{* *}$ \\
\hline 8 Positive affect & $\begin{array}{l}3.83 \\
(0.82)\end{array}$ & $\begin{array}{l}3.90 \\
(0.76)\end{array}$ & .02 & $.65^{* * *}$ & $.57 * * *$ & $-.18^{*}$ & $-.29 * * *$ & $-.24 * *$ & $-.48 * * *$ & & $-.39 * * *$ \\
\hline 9 Negative affect & $\begin{array}{l}2.04^{b} \\
(0.93)\end{array}$ & $\begin{array}{l}1.79^{b} \\
(0.65)\end{array}$ & -.04 & $-.48^{* * *}$ & $-.56 * * *$ & .15 & $.36 * * *$ & $.34 * * *$ & $.52 * * *$ & $-.63 * * *$ & \\
\hline
\end{tabular}

Note. Girls $n=187$, boys $n=157$. Correlation coefficients above the diagonal relate to boys and coefficients below the diagonal relate to girls. ${ }^{*} p<.05,{ }^{* *} p<.01,{ }^{* * *} p<.001$. Gender difference in variables indicated a $p=.05,{ }^{b} p<.01,{ }^{c} p<.001$. 